\title{
What Does the Frontomedian Cortex Contribute to Language Processing: Coherence or Theory of Mind?
}

\author{
Evelyn C. Ferstl ${ }^{1}$ and D. Y ves von Cramon \\ Max Planck Institute of Cognitive Neuroscience, Stephanstrasse 1a, D-04103, Leipzig, Germany
}

Received December 20, 2001

The frontomedian cortex (F MC) has been shown to be important for coherence processes in language comprehension, i.e., for establishing the pragmatic connection between successively presented sentences. The same brain region has a role during theory-ofmind processes, i.e., during the attribution of other people's actions to their motivations, beliefs, or emotions. In this study, we used event-related functional magnetic resonance imaging at $3 \mathrm{~T}$ to disentangle the relative contributions of the FMC to theory-of-mind (ToM) and coherence processes, respectively. The BOLD response of nine participants was recorded while they listened to pragmatically coherent or unrelated sentence pairs. Using a logic instruction for inanimate sentence pairs, ToM processing was discouraged during the first part of the experiment. Using explicit ToM instructions for sentence pairs mentioning human protagonists, ToM processing was induced during the second part. In three of the resulting four conditions a significant increase in the BOLD response was observed in FMC: when ToM instructions were given, both coherent and incoherent trials elicited frontomedian activation, in replication of previous results showing involvement of the FMC during ToM tasks. When logic instructions were given, the coherent trials, but not the incoherent trials, activated the FMC. These results clearly show that the FMC plays a role in coherence processes even in the absence of concomitant ToM processes. The findings support the view of this cortex having a domain-independent functionality related to volitional aspects of the initiation and maintenance of nonautomatic cognitive processes. 2002 Elsevier Science (USA)

Key Words: coherence; theory of mind; functional magnetic resonance imaging; frontomedian cortex; text comprehension.

${ }^{1}$ To whom correspondence should be addressed. Fax: ++49 (341) 9940 221. E-mail: ferstl@ens.mpg.de.

\section{INTRODUCTION}

For social interaction, the ability to understand other people's beliefs, motivations, and goals is crucial. "Mentalizing" (Frith and Frith, 1999) or Theory of Mind (Premack and Woodruff, 1978), as this ability has been termed, is at the core of successful interaction with other human beings. Theory of Mind (TOM) is necessary for understanding and predicting other people's behavior and, thus, for reacting adaptively to the changing environment. There is a large body of knowledge about the development of this faculty in childhood (Wellman, 1993), and it has been shown that autistic children have specific deficits in ToM processing (Baron-Cohen et al., 1985). Recently, a number of neuropsychological patient studies and neuroimaging studies have attempted to further our understanding of the neuroanatomical realizations of ToM abilities. A cortical region shown to be particularly important for ToM processes is the frontomedian cortex (FMC; also termed the dorsal medial prefrontal cortex), comprising mainly the medial aspects of Brodmann area (BAs) 9 and reaching into the adjacent portions of BAs 8, 10, and 32. In the following we first review the evidence for the FMC being involved during ToM processing, and then raise the question of whether other, more general processes might engage the FMC as well. Finally, we present an experiment designed to explicitly test the hypothesis that ToM processes are sufficient, but not necessary for FMC activation.

Patient studies on ToM deficits after acquired brain injury include investigations of right hemisphere involvement (Happé et al., 1999; but see Siegal et al., 1996) and the role of the amygdala (Fine et al., 2001). Because most tasks employed for testing ToM abilities involve complex reasoning and working memory processes, on one hand, or the processing of affective information, on the other, a number of studies have focused on the effect of lateral prefrontal and orbitofrontal lesions on ToM abilities. Channon and Crawford (2000) found that left frontal lesions were most likely to cause a deficit in a story comprehension task involving ToM processes. Similarly, Rowe et al. 
(2001) documented impairments in a false belief story comprehension task. Indpendent of laterality, most patients in their sample had lesions including medial or orbitofrontal regions. Additional evidence for the importance of medial, rather than dorsolateral frontal areas for ToM was provided by Stone et al. (1998), using a faux pas task, and by Stuss et al. (2001), using a deception task. Taken together, these few studies provide strong evidence for a frontal, and particularly a frontomedian, contribution to ToM abilities.

Additional evidence for the involvement of frontal brain areas is provided by a growing number of PET and fMRI studies on ToM processes. Whereas most patient studies used second- and third-order belief tasks or deception tasks, i.e., tasks whose properties are well known from the developmental literature on ToM processing, the imaging studies employed a set of different tasks. A most influential paradigm was introduced by Fletcher et al. (1995). In their PET study, they directly compared the processing of stories matched in length and complexity, but different with respect to an inherent ToM component. When ToM processing was required, the anterior and posterior cingulate cortices, a right inferior parietal region, and, most importantly, a region in the frontomedian wall (BA 8/9) were activated. This finding has been replicated with $\mathrm{fMRI}$ imaging, with visual stimuli (cartoon stories) instead of language stimuli (Gallagher et al., 2000), and with stories worded in first person rather than in third person (Vogeley et al., 2001).

Other studies using paradigms intended to induce different aspects of ToM processing yielded FMC activation as well. In a face perception study, a large, unspecific network was shown to be involved when the affective expression was to be judged, as compared with the person's gender (Baron-Cohen et al., 1999); in an object perception study, more activation was found in FMC when an inference on other people's beliefs about the objects was required, compared with the retrieval of the objects' use from semantic memory. And finally, the perceived intentionality or goal orientation of visually presented stimuli predicted the FMC activation, both for meaningful cartoon stories (Brunet et al., 2000) and for abstract movement patterns (Castelli et al., 2001). Even for autistic or Asperger's patients, whose ToM abilities seem impaired, some F MC activation was reported during different ToM tasks (Baron-Cohen et al., 1999; Happé et al., 1996).

These imaging results clearly confirm that, among other brain regions, the FMC is part of the "social brain" (Brothers, 1990). However, evidence against the view of FMC being specific for ToM, i.e., against the view that ToM processes are necessary for activating the FMC, comes from imaging studies reporting FMC activation elicited by tasks other than ToM tasks. For instance, Zysset et al. (2002) reported FMC activation for the judgment of evaluative statements, Goel et al.

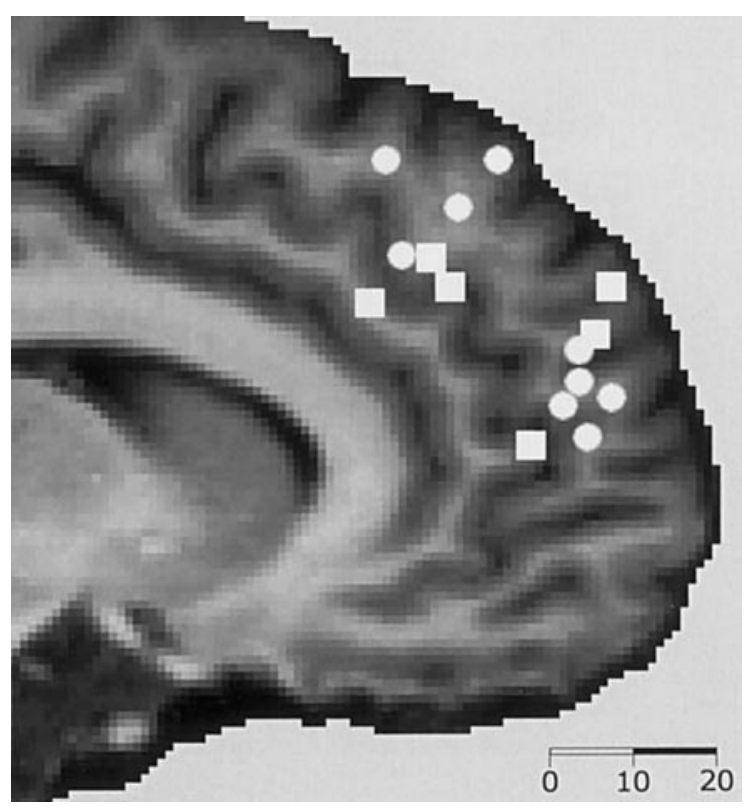

FIG. 1. Frontomedian peaks of activation from selected imaging studies (restricted to BA 8-10, irrespective of lateralization). The squares are from ToM tasks (Castelli et al., 2000; Fletcher et al., 1995; Gallagher et al., 2000; Goel et al., 1995; Vogeley et al., 2001), the circles denote peaks from plausibility judgments, reasoning, evaluation, coherence judgment, and self-referential processing (Bottini et al., 1994; Ferstl and von Cramon, 2001; Goel et al., 1997; Greene et al., 2001; Gusnard et al., 2001; Zysset et al., 2002). It is apparent that both types of tasks activate similar regions in FMC.

(1997) for inductive as compared with deductive reasoning, and Gusnard et al. (2001) for self-referential judgments compared with neutral classifications. A recent study on moral judgment argued that the same pattern of frontomedian and retrosplenial activation is caused by emotional processing (Greene et al., 2001). Another group of studies concerned higher-level Ianguage comprehension. FMC activation was found in the context of pragmatic comprehension (Bottini et al., 1994; Nichelli et al., 1995) and story comprehension (Maguire et al., 1999).

To illustrate how well the results of these studies overlap, despite the apparent variety of tasks employed, Fig. 1 displays the peaks of the activated regions for a selection of studies, projected onto the left median wall of an individual brain. As can be seen, there is no obvious anatomical division between studies investigating ToM processes, on one hand, and studies on plausibility, language, or evaluative judgments, on the other.

To illustrate two possible explanations for this overIap, let us consider a previous fMRI study on inference processes during text comprehension. Presented with sentence pairs (see Table 1), the participants' task was to evaluate their coherence (cf. Kintsch, 1998), i.e., to judge whether there was a pragmatic connection be- 
TABLE 1

Sentence Examples

Coherent

Incoherent

Part A: Logic

Part B: Theory-of-Mind
Sometimes a truck drives by the house.

That's when the dishes start to rattle.

The lights have been on since last night.

The car doesn't start.

Mary's exam was about to begin.

Her palms were sweaty.
Sometimes a truck drives by the house.

The car doesn't start.

The lights have been on since last night.

That's when the dishes start to rattle.

Mary's exam was about to begin.

Some friends had remembered the birthday.

Laura got a lot of mail today.

Her palms were sweaty.

Laura got a lot of mail today.

Some friends had remembered the birthday.

tween the two sentences or not. ${ }^{2}$ During performance of this task, complex inference processes are carried out. It is necessary to comprehend both sentences, to store the context sentence's content in working memory, to retrieve relevant world knowledge, and finally to integrate all these information sources with the information provided in the second sentence. In contrast to the expectation that the perisylvian language areas, or their right hemispheric homologues, are active mainly during inferencing, we found medial cortical regions to be sensitive to coherence. Specifically, the FMC and a corresponding posterior cingulate re gion (PCC) were more active during the processing of coherent as compared with incoherent trials (Ferstl and von Cramon, 2001).

The first explanation for overlapping FMC activation is that in the studies on evaluative, reasoning or coherence processes, concurrent ToM processes were inadvertently induced, although the tasks were not explicitly designed for doing so. For instance, tasks in which the participants' opinions are asked for, such as those employed in the Gusnard and Zysset studies, can belinked to ToM processing under the assumption that understanding other people's minds is closely related to reflecting on one's own (Vogeley et al., 2001). Any task in which the plausibility of statements needs to be evaluated, in particular in the context of human protagonists, might automatically elicit ToM processes. In our own materials for the coherence judgment task (Ferstl and von Cramon, 2001), sentence pairs with and without human protagonists were intermixed. For the animate sentence pairs (shown in the bottom rows of Table 1), the coherence judgments could have been based on a ToM evaluation. Rather than answering the question: "Can I find a pragmatic connection between

\footnotetext{
${ }^{2} \mathrm{~N}$ ote that we use the term coherence in its linguistic sense only. The related concept of central coherence used in autism research is considerably more general (Frith, 1989; see Happé, 2000, for a review), although it encompasses language processes (cf. J olliffe and Baron-Cohen, 1999, 2000).
}

these two sentences?" subjects might simply have answered the question: "Can I understand what these people are doing?"

A second explanation of why coherence and ToM processes both elicit FMC activation is that this cortex might subserve a more general function concomitant with both coherence and ToM processing. Gusnard et al. (2001), for instance, interpreted the BA 8/9 activation as being related to self-referential processes (cf. Vogeley et al., 2001), and Greene et al. (2001), as related to emotional processes. In our own study, we interpreted the FMC activation as being related to the initiation and maintenance of nonautomatic cognitive processes (Ferstl and von Cramon, 2001), in particular when long-term memory and evaluative components are needed for task performance (Zysset et al., 2002).

The present study was designed to provide evidence for the second explanation. The goal was to prove ToM processing to be sufficient, but not necessary for activating the FMC. The study had two event-related parts ( $A$ and $B$ ) in each of which coherent and incoherent trials were intermixed. Part A was designed to show that coherence alone could activate the FMC, even in the absence of ToM processes: we first selected the inanimate sentence pairs from our previous study (Ferstl and von Cramon, 2001) for separate presentation (Part A). To discourage ToM processes, the instructions focused on the internal logic of the sentence pairs. Part B was designed to show that ToM alone could activate the FMC, in the absence of coherence. During Part B, the animate sentence pairs were presented. To induce ToM processing, the participants were asked to try to identify with the protagonists mentioned, and to attempt to understand their motivations for and feelings during the actions described. Since the crucial comparison concerned the replication of the coherence effect in the absence of concurrent ToM processes, Part A was always presented first.

The predictions are clear-cut. If in FMC a specific ToM process was realized, its activation would be ex- 
pected during Part B only, but not during Part A. If, in contrast, the frontomedian activation was due to coherence processes, FMC activation would be expected for coherent trials, during Part A as well as during Part B, but not for incoherent trials. If, as we believe, both processes share a more general, domain-independent component related to the initiation and maintenance of nonautomatic cognitive processes, we expect a replication of the coherence effect during Part $A$, as well as FMC activation for both coherent and incoherent trials during Part $\mathrm{B}$.

\section{METHODS}

\section{Participants}

Five women and four men, all right-handers, received reimbursement for participating in the experiment. None of the participants had any history of neurological disorder or other health problems preventing them from being exposed to the magnetic field. The median age was 24 years (range 22-27). All participants had given informed consent.

\section{Design and Materials}

The experiment consisted of two independent parts ( $A$ and $B$ ) with identical procedure. In both parts, the within-subjects factor Coherence (coherent vs incoherent) was manipulated, but the types of materials as well as the instructions were varied. In Part A (Logic) ToM processes were minimized, whereas in Part B (ToM) ToM processes were explicitly induced. The materials were taken from the previous fMRI study (Ferstl and von Cramon, 2001). The language trials were made up of 120 coherent sentence pairs in which the second sentence (the target) was pragmatically related to the first (the context). For Part A, those 60 sentence pairs were selected that referred to objects only and did not mention human protagonists. For Part $B$, those 60 sentence pairs were used that contained references to people. The incoherent conditions were created by switching the context sentences of two coherent trials. Examples for the resulting four types of trials are provided in Table $1 .^{3}$

All sentences were read by a female speaker and tape recorded. By separating the recording of the context sentences and the target sentences, prosodic cues as to the coherence of the trials were avoided. F or the computer presentation the recordings were digitalized and their loudness was adjusted.

The presentation length for the sentence pairs was $5.3 \mathrm{~s}$ on average $(\mathrm{SD}=0.7$, range $=3.0-8.1 \mathrm{~s})$, and

\footnotetext{
${ }^{3}$ As in our previous experiment (Ferstl and von Cramon, 2001), half of the target sentences in each condition contained cohesive ties, i.e., words explicitly signaling a connection between the sentences, such as pronouns and conjunctions.
}

there were no differences as a function of Part or Coherence (all $F$ 's $<1$ ).

For the control condition in the fMRI experiment, we used an additional 32 trials made up of pseudo-word sentences. Because it was necessary to control for both the auditory perception and the task demands (listening to the two "sentences," relating them to each other, and providing a motor response), we used two different types of pseudo-sentences. Half of the pseudo-sentences contained some German function words or function morphemes (e.g., Der Miefensalm ist noch kolmut geklubet), and half did not (Molsa erkau lanschdal ettenbul giller). Thirty-two pseudo-sentence pairs were then created so that in 16 of the trials, the two pseudosentences were from the same item type (consistent), and in the other 16 they were from different item types (inconsistent). The length of the control trials was comparable to that of the experimental trials ( $\mathrm{M}=5.4 \mathrm{~s}$, range $=3.6-6.7 \mathrm{~s}, \mathrm{SD}=0.8$ )

For the event-related experiment, we intermixed stimuli from the three conditions (coherent, incoherent, control) as follows: For both Part A and Part B separately, four counterbalancing lists of the 60 experimental items were created. Within each list, 30 target sentences were paired with their coherent context sentence; the remaining 30 target sentences were paired with the incoherent context. Across the four lists, each sentence appeared twice in each of the two conditions. The 16 control trials were intermixed with the experimental trials. Once more, half of the control trials were in each of the two consistency conditions. The order of the trials in each of the resulting lists was randomized separately, with the constraint of not more than three consecutive trials being in the same condition. In each of the two experiment parts, a warmup trial at the beginning and a buffer trial at the end were added. Thus, the two parts of the experiment consisted of a total of 78 trials each.

\section{Procedure}

The participants received written instructions for Part A (Logic) before the scanning session started. The participants were told to carefully listen to the sentence pairs and to indicate after the second sentence whether there was a logical connection to the first sentence or not. Two examples each were given for coherent and incoherent sentence pairs and the appropriate response (YES or NO) was indicated. Furthermore, the participants were informed that trials with nonword sentences would be interspersed. Examples were given for the two types of stimuli. The participants were told that they were from different artificial languages, one of which sounded somewhat like German while the other had no resemblance to German. The task for these trials was to indicate whether the pseudo-language was the same or different for the two 
nonword sentences. For their YES responses, half of the participants were instructed to use the key on the right side of the response box, and the other half to use the key on the left.

During the scanning session, the participants lay flat inside the magnet, with a response box placed into their right hands. They were instructed to keep their eyes closed throughout the experiment. The stimuli were presented to both ears through headphones. To protect from the scanner noise, participants wore ear plugs that muffled the noise while allowing the stimuli to be heard.

The functional measurement for Part A involved the presentation of one of the four lists of inanimate sentence pairs. Each trial lasted $20 \mathrm{~s}$, with the following time course: A short beep alerted the subject to the beginning of the presentation 500 ms before the first sentence was played. Then the two sentences were presented with an interstimulus interval of $500 \mathrm{~ms}$. The timing of the trial was such that the onset of the second sentence always occurred $6200 \mathrm{~ms}$ after the trigger signaling the beginning of the trial. This timing yielded variable intertrial intervals, whose length depended on the response time of the previous trial and the duration of the context sentence of the current trial. The reaction times were measured from the onset of the target sentence up to the participant's button press for the YES or NO response. There was an unfilled pause until the next presentation started.

The instructions for the second part of the experiment (Part B: ToM) were read to the participants after the first part had been completed. Remaining in the scanner, the participants were informed that the subsequent sentences would refer to human protagonists. The instructions further stated (translated from German): "Your task is to identify with the people mentioned. You should try to put yourself into their shoes, i.e., to understand their motivations, feelings, and actions. After the second sentence, please press the YES key if you succeeded, and the NO key if you did not. It is especially important to take into account both sentences." Furthermore, the instructions stated that the control task was continued in the same way as in the first part of the experiment. After the experimenter had ensured that these instructions were understood, functional measurement for Part B started. The 78 animate trials were presented with a time course identical to that of Part A.

The presentation of the two parts of the experiment lasted $52 \mathrm{~min}$, and the entire scanning session had a duration of about 65-70 min.

\section{Data Acquisition}

A Bruker Medspec 30/100 system was used for magnetic resonance imaging at 3.0 T. Prior to the functional scans, two anatomical scans were acquired for each participant using MDEFT sequences (Ugurbil et al., 1993). The first was a whole-brain image acquired with a T1-weighted 3D segmented sequence (Norris, 2000; 128 sagittal, adjacent slices, $1.5 \mathrm{~mm}$ thick, $256 \times$ 256-pixel matrix per slice; TR $1.3 \mathrm{~s}$, TE $10 \mathrm{~ms}$ ). To enable alignment of the functional scans with this high-resolution image, anatomical T1-weighted 2D images were acquired, using the same number and orientation of slices as the functional scans.

During the functional scans, the BOLD response was measured using a single-shot gradient EPI sequence (matrix $64 \times 64, \mathrm{TE}=30 \mathrm{~ms}$, flip angle $90^{\circ}$, field of view $192 \mathrm{~mm}$, acquisition bandwidth $100 \mathrm{kHz}$ ). Horizontal images were acquired for 16 slices (5-mm thickness, 2-mm spacing), parallel to the bicommissural plane (AC-PC). For most participants, 6 slices were bel ow the AC-PC line, while 10 slices were above, but care was taken that the temporal lobes, as well as prefrontal regions, were covered in full. In-plane resolution was $3 \times 3 \mathrm{~mm}$. We used a repetition time of $2 \mathrm{~s}$ $(T R=2)$, and the presentation of the displays was triggered by the acquisition of the first slice of the current image.

\section{Data Analysis}

Data analysis was conducted using the software package LIPSIA, developed as a tool for analyzing functional MRI data (Lohmann et al., 2001).

For each participant, the signal acquired during the functional scans was preprocessed as follows: First, a sinc-interpolation algorithm was applied to correct for the temporal spacing between the 16 slices of each image. Motion correction consisted of a global affine linear transformation that optimized for each time step the linear correlation between the image at that time step and a predefined reference image. A baseline correction was then conducted using a temporal high-pass filter with a cutoff frequency of $1 / 40 \mathrm{~Hz}$. Furthermore, a spatial Gaussian filter was applied with a standard deviation of $\sigma=0.8$. With a voxel size of $3 \mathrm{~mm}$, this standard deviation is equivalent to a FWHM of 5.65.

For the statistical analysis we carried out the following sequence of processing steps (Lohmann et al., 2001): For each participant separately, the 2D data were analyzed using the General Linear Model (cf. Friston, 1994) based on a 3 (Trial: coherent, incoherent, control $) \times 2$ (Part A, Part B) within-subject design. For the event-related model we time-locked the BOLD responses $1000 \mathrm{~ms}$ before the offset of the target sentence. A synthetic hemodynamic response function with a lag of $6 \mathrm{~s}$ was assumed. The model equation, i.e., the observation data, the design matrix, and the error term, was convolved with a Gaussian kernel with a dispersion of $4 \mathrm{~s}$ FWHM. Contrast codes were then used to detect significant activations by calculating $t$ statistics based on the parameter estimates of the full 
linear model, and subsequently transforming the t values into $Z$ scores. For each participant, the following five comparisons were made: For each part separately all language trials were compared with the control trials, and then the coherent with the incoherent trials. Because of the fixed order of the two parts of the experiment, it was not feasible to directly compare the activations in the two parts. However, to statistically strengthen the resulting differences, some results from the interaction contrasts between Language and Part, as well as Coherence and Part, are also reported.

F or the statistical analysis across participants, all Z maps of each participant were fitted into a standard stereotaxic space (Talairach and Tournoux, 1988) as follows: First, the 2D anatomical scan was rotated and shifted so that it mapped onto the 3D whole-brain image. Then, linear scaling factors were calculated to transform the image to the standard size. The resulting coregistration matrices were finally applied to the contrast images using trilinear interpolation (Lohmann, 1998). The resulting standardized 3D images were finally transformed using deformation fields obtained from a nonlinear adjustment of the participants' individual anatomies (Thirion, 1998). The resulting $Z$ maps were then averaged across participants (Bosch, 2000). Only those voxels with $|Z|>3.09$ were considered significantly activated $(P<0.001)$. Since this probability level is uncorrected for multiple comparisons, we defined an additional spatial extent threshold. Areas of activation smaller than $225 \mathrm{~mm}^{3}$ were neglected. This threshold roughly corresponds to the requirement that at least 5 adjacent voxels in the original image (of volume $3 \times 3 \times 5 \mathrm{~mm}=45 \mathrm{~mm}^{3}$ ) be significantly activated.

F or the displays of the results, the deformation fields from the nonlinear scaling were applied to the individual participants' anatomical images, and a mean brain was obtained by averaging these normalized images. In all figures, the $Z$ maps are overlaid on this mean brain for our group of participants.

\section{RESULTS}

\section{Behavioral Data}

For a measure of performance, the proportions of answers coinciding with the coherence of the sentence pair were calculated. A $2 \times 2$ ANOVA with the factors Part and Coherence yielded a main effect of Part $(F(1,8)=10.1, P<0.01)$. During the first part, only $7.6 \%$ (SD $=3.3$ ) of the responses deviated from those predicted by Coherence, whereas in the second part $18.7 \%(S D=11.4)$ of the responses deviated. Although neither the main effect of Coherence nor the interaction between Coherence and Part reached significance $(F(1,8)=2.2$ and $F(1,8)<1.0$, respectively), inspection of the data showed that this was due to large intersub-

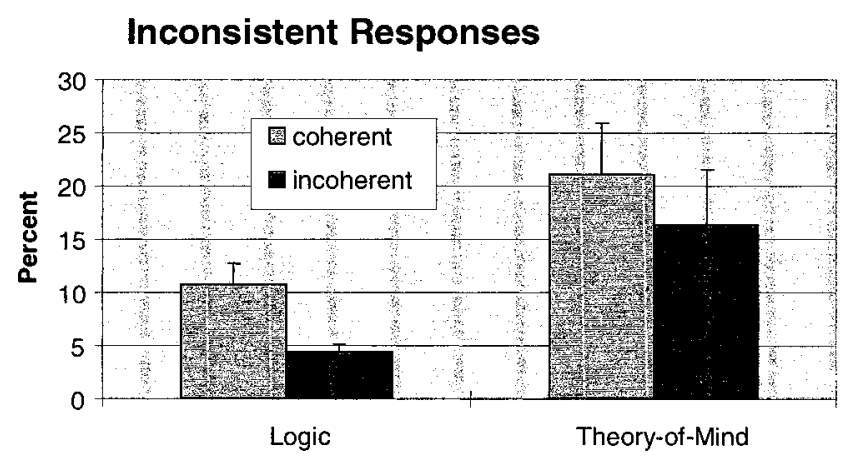

Reaction Times

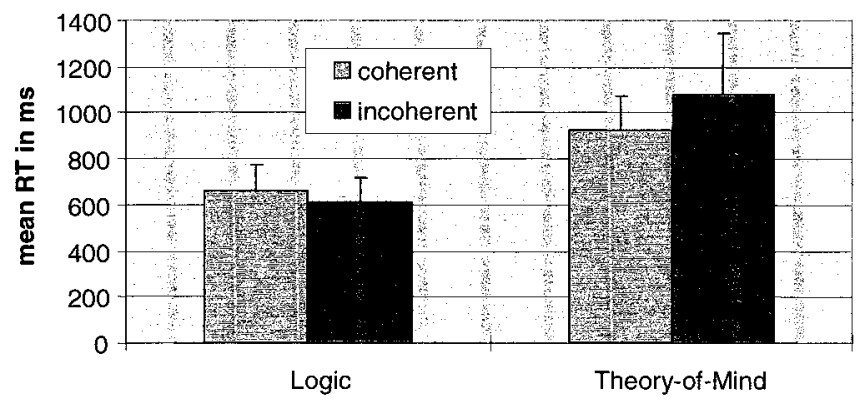

FIG. 2. Behavioral data for the two parts of the experiment. Top: Mean percentage of the responses coinciding with the coherence of the sentence pair. Bottom: Mean response times. The error bars are one standard error above the mean.

ject variability during Part B. Planned pairwise comparisons confirmed that Coherence had an effect during Part $A(F(1,8)=9.6, P<0.02)$. Slightly more answers deviated for the coherent trials than for the incoherent trials. No such effect was observed during Part B $(F(1,8)<1)$.

For an analysis of the response times, measured from the onset of the target sentences, the presentation duration of the target sentences was subtracted. The resulting reaction times correspond to the latency of the response after the offset of the target sentence. The means of the response times are shown in Fig. 2 for both parts as a function of coherence. The overall mean reaction time was $818 \mathrm{~ms}(\mathrm{SD}=408)$ and varied from $400 \mathrm{~ms}$ up to $1700 \mathrm{~ms}$. To control for this large variance, the reaction times were first standardized for each subject separately. The statistical analysis, based on the resulting $Z$ scores, yielded a main effect of Part $(F(1,8)=7.4, P<0.05)$. The reaction times were longer during Part $B$ than during Part $A$. Neither the main effect of Coherence $(F(1,8)<1)$, nor the interaction with Part $(F(1,8)=2.4, P=0.15)$ reached significance.

These results confirm that the participants heeded the instructions. In both performance and reaction times, there were clear effects of the experimental task. Furthermore, the response pattern during Part $A$ 
TABLE 2

Characteristics of the Cortical Regions of Activation for the Contrast Comparing All Language Trials with the Control Task ${ }^{\mathrm{a}}$

\begin{tabular}{|c|c|c|c|c|c|c|c|c|c|c|c|c|}
\hline \multirow[b]{2}{*}{ Language-Control } & \multirow[b]{2}{*}{ BA } & \multirow[b]{2}{*}{ Side } & \multicolumn{5}{|c|}{ Part A: Logic } & \multicolumn{5}{|c|}{ Part B: Theory of Mind } \\
\hline & & & Size & $Z_{\max }$ & $x$ & Y & z & Size & $Z_{\max }$ & $x$ & Y & $\mathrm{Z}$ \\
\hline 1. Frontomedian cortex & $9 / 10 / 32$ & $\mathrm{~L}$ & 960 & 5.32 & -16 & 51 & 30 & 7,548 & 6.57 & -19 & 49 & 30 \\
\hline \multirow{2}{*}{ 2. Retrosplenial cortex/precuneus } & 29/30/23/31 & L & 1,745 & 6.66 & -11 & -59 & 15 & 17,946 & 9.10 & -10 & -58 & 21 \\
\hline & $29 / 30$ & $\mathrm{R}$ & 608 & 5.39 & 8 & -57 & 14 & - & - & 6 & -55 & 12 \\
\hline 3. Temporoparietal cortex & $39 / 22$ & L & 8,541 & 7.71 & -43 & -63 & 27 & 10,624 & 9.11 & -50 & -65 & 25 \\
\hline 4. Posterior STS & $22 / 21$ & L & - & - & -60 & -58 & 7 & 869 & 5.66 & -59 & -58 & 8 \\
\hline \multirow[t]{2}{*}{ 5. Anterior STS } & 21 & L & 4,314 & 8.26 & -53 & -6 & -13 & 5,806 & 8.56 & -54 & -13 & -8 \\
\hline & 21 & $\mathrm{R}$ & 1,682 & 6.47 & 45 & -2 & -16 & 3,767 & 8.27 & 46 & -2 & -16 \\
\hline \multirow[t]{2}{*}{ 6. Fusiform/parahippocampal gyrus } & $35 / 36$ & $\mathrm{~L}$ & 1,491 & 5.88 & -33 & -34 & -9 & 1,002 & 5.49 & -31 & -33 & -9 \\
\hline & $35 / 36$ & $\mathrm{R}$ & & & & & & 296 & 4.41 & 28 & -25 & -12 \\
\hline 7. Superior frontal sulcus & 8 & L & & & & & & 1,179 & 5.24 & -19 & 29 & 48 \\
\hline 8. Inferior frontal gyrus & 45 & L & & & & & & 349 & 4.79 & -48 & 17 & 0 \\
\hline 9. Lateral occipital gyri & 19 & $\mathrm{R}$ & & & & & & 793 & 4.33 & 29 & -93 & 13 \\
\hline 10. Parieto-occipital cortex & $39 / 19$ & $\mathrm{R}$ & & & & & & 537 & 4.65 & 41 & -77 & 12 \\
\hline
\end{tabular}

${ }^{a}$ Shown are the approximate Brodmann areas, $\mathrm{Z}$ values, and Talairach coordinates for the peaks and the size of the region of activation (in $\mathrm{mm}^{3}$ ). The activations are displayed in Fig. 3, using the same labels. The thresholds were set to Z $>3.8$ and a minimal size of the regions of $225 \mathrm{~mm}^{3}$.

closely resembled that of the previous experiments using a coherence judgment task.

\section{fMRI Results}

For Part A and Part B separately, Table 2 provides a list of the significant regions of activation from the contrast comparing all language trials with the control task. To focus the discussion, only the most prominent areas are listed $\left(\mathrm{Z}>3.8, \mathrm{P}<.0001\right.$; extent $\left.>225 \mathrm{~mm}^{3}\right)$.

Figure 3 illustrates that remarkably similar networks were engaged in both parts of the experiment, with Part B yielding stronger activations than Part A. As expected, language processing in context involved the anterior portion of the superior temporal sulcus bilaterally (BA 21), more pronounced in the left hemisphere. In both parts, there was activation along the collateral sulcus (fusiform/parahippocampal gyri) on the left side, with the corresponding region on the right being significant during the ToM part only. In both parts, there was strong involvement of a temporoparietal region (BA 39/22), including the angular gyrus. Three additional, small regions reached the threshold during Part $B$ only: the tip of the triangular part of the inferior frontal gyrus (BA 45), a region in the parietooccipital transition area (BA 39/19), and one in the lateral occipital gyri (BA 19).

In the median wall, there were two large regions of activation. The center of gravity of the bilaterally active posterior region lay in the retrosplenial area (BA 29/30). In Part B, this activation extended further into the left inferior precuneus (BA 23/31). The anterior border was delineated by the marginal branch of the cingulate sulcus, the posterior border by the "common stem" and the parieto-occipital sulcus. The frontal region, clearly left lateralized, fell into the median part of the superior frontal gyrus, dorsal to the paracingulate sulcus (posterior BA 9). In Part B, this activation extended ventrally into BA 10 and dorsally into the superior margin (BA 9/10/32), and it was accompanied by a morelateral, small area in the superior frontal sulcus (BA 8).

The most apparent difference between the activation patterns for the two parts of the experiment was that the median regions were more strongly activated during Part B than during Part A. To statistically strengthen the different involvement of median structures during the two parts, we calculated the contrast coding the interaction between the effects of language and the effects of part. In confirmation of the descriptive differences, both the posterior $\left(Z_{\max }=5.73\right.$; size $=$ $18,595 \mathrm{~mm}^{3}$; peak [1 -66 38]), as well as the anterior median region $\left(Z_{\max }=4.51\right.$; size $=2299 \mathrm{~mm}^{3}$; peak $[-4$ 52 10]) proved to be more active during Part $B$ than during Part $A$.

The results for the contrast comparing coherent with incoherent trials is shown in Table 3. F or Part B, under the ToM instructions, this contrast did not yield any significant regions of activation, indicating that coherent and incoherent trials were processed in a similar fashion. During Part A, using the Logic instructions, the results resembled those of our previous study (Ferstl and von Cramon, 2001): None of the regions were more active during incoherent trials than during coherent trials. For the reverse comparison, however, 


\section{Language-Control}

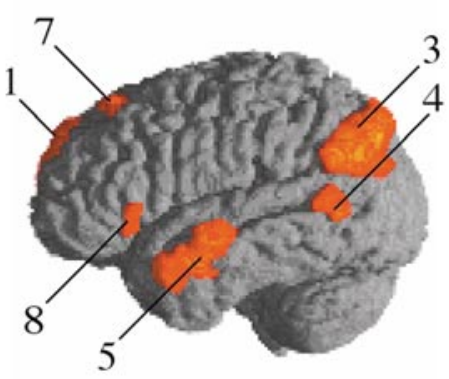

Theory-of-Mind
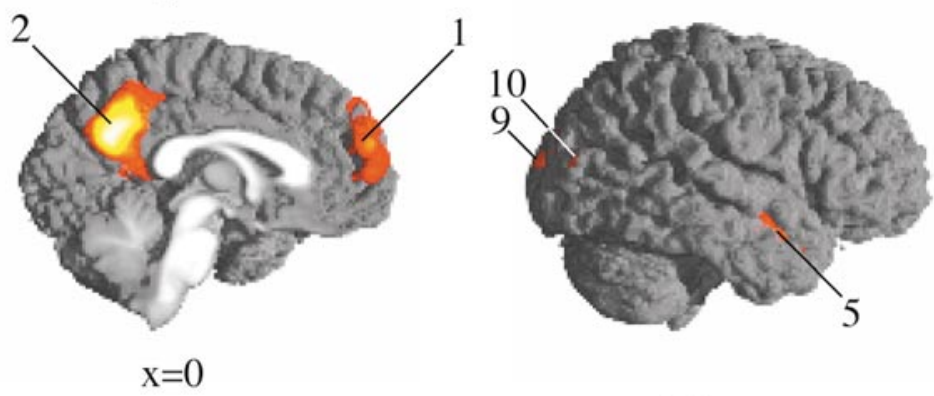

$z>3.8$

Logic
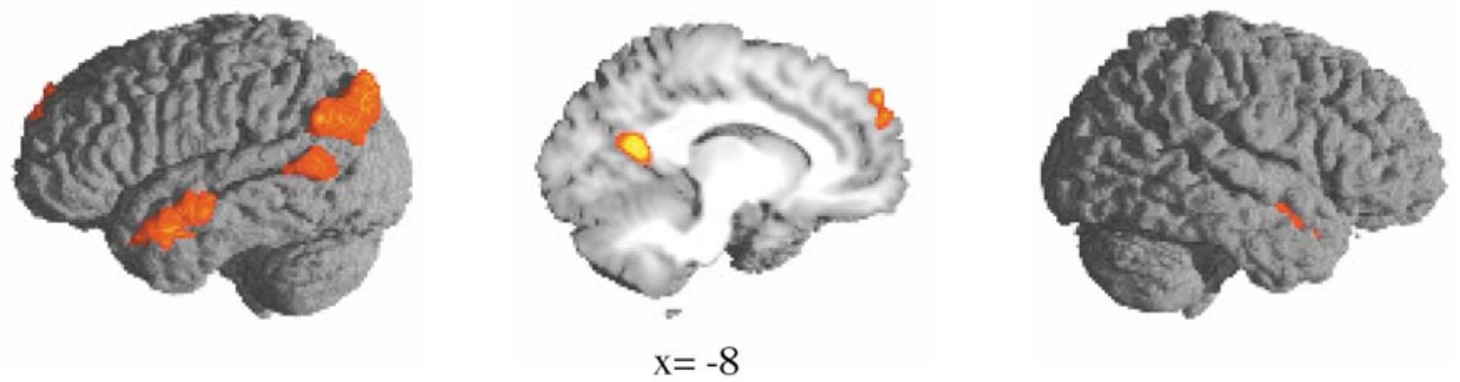

\section{Logic: Coherent-Incoherent}

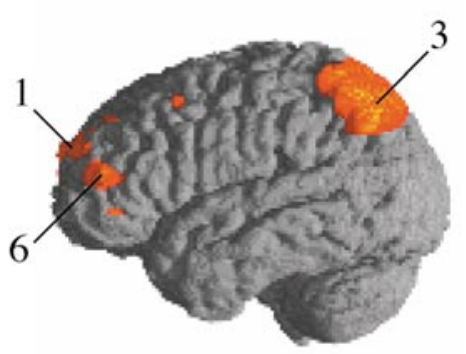

L

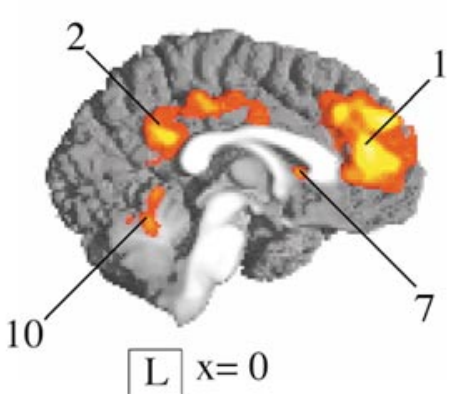

L $x=0$

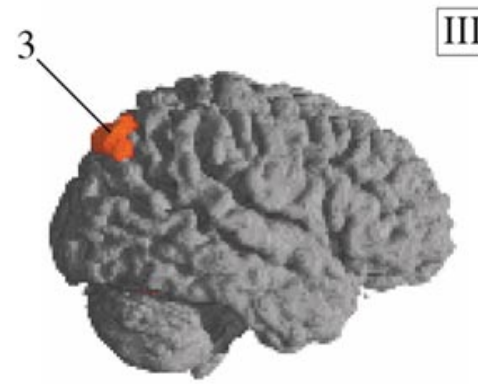

$\mathrm{z}>3.1$ 
TABLE 3

Cortical Regions of Activation for the Contrast Comparing Coherent with Incoherent Trials $\left(Z>3.09 \text {, Size }>225 \mathrm{~mm}^{3}\right)^{\mathrm{a}}$

\begin{tabular}{|c|c|c|c|c|c|c|c|}
\hline \multirow[b]{2}{*}{ Coherent-Incoherent } & \multirow[b]{2}{*}{ BA } & \multirow[b]{2}{*}{ Side } & \multicolumn{5}{|c|}{ Part A: Logic } \\
\hline & & & Size & $Z_{\max }$ & $x$ & Y & Z \\
\hline 1. Frontomedian cortex/ACC & 9/10/24/32 & $\mathrm{L}$ & 24005 & 5.57 & -6 & 26 & 35 \\
\hline 2. Retrosplenial area/PCC & $29 / 30 / 23$ & L & 6281 & 4.51 & -4 & -28 & 35 \\
\hline \multirow{2}{*}{ 3. Posterior parietal cortex } & 39 & L & 9350 & 4.98 & -54 & -62 & 41 \\
\hline & 39 & $\mathrm{R}$ & 1705 & 4.05 & 43 & -70 & 44 \\
\hline 4. Inferior precentral sulcus & $6 / 44$ & L & 753 & 4.24 & -36 & -2 & 25 \\
\hline 5. Superior frontal sulcus & 10 & $\mathrm{~L}$ & 374 & 4.15 & -27 & 34 & 34 \\
\hline 6. Inferior frontal gyrus & $45 / 46 / 47$ & L & 1393 & 4.10 & -45 & 33 & 1 \\
\hline 7. Caudate nucleus & - & $\bar{L}$ & 7106 & 4.66 & -13 & 3 & 18 \\
\hline 8. Midbrain (VTA) & - & L & 873 & 4.90 & -8 & -20 & -6 \\
\hline 9. Hippocampal formation & - & L & 691 & 4.19 & -25 & -26 & -6 \\
\hline 10. Cerebellum & - & $\mathrm{L}$ & 1433 & 3.72 & -3 & -53 & -15 \\
\hline 11. Cerebellum & - & $\mathrm{R}$ & 978 & 3.85 & 32 & -58 & -20 \\
\hline
\end{tabular}

\footnotetext{
${ }^{a}$ There were no significant results for the comparison of incoherent with coherent trials. During Part B (Theory of Mind), the two conditions did not yield any different regions of activation either.
}

fundus of the left inferior precentral sulcus (BA 44/6), proved to be more active during coherent trials than during incoherent trials. ${ }^{4}$

For further confirmation of the differential effect of Coherence in the median wall of the left hemisphere during the two parts of the experiment, the contrast coding the interaction between Coherence and Part was calculated. And indeed, this contrast yielded two regions in the cingulate sulcus that were significantly more sensitive to coherence during Part $A$ than during Part B (BA 8/9/32, [ - $\left.\begin{array}{lll}-2 & 25 & 35\end{array}\right], Z_{\max }=4.59$, size $=4498$ $\mathrm{mm}^{3}$; and $\mathrm{BA} 9 / 10 / 32$ [-4 3916$], Z_{\max }=3.8$, size $=668$ $\mathrm{mm}^{3}$ ). In contrast to these FMC activations, the coherence effect in the PCC/precuneus did not significantly differ for the two parts of the experiment.

\section{Region-of-Interest Analysis}

To evaluate which of the observed differences provided by the statistical comparisons was due to activation of the relevant areas, rather than to deactivation in one of the comparison conditions, the frontomedian changes in the BOLD contrast were analyzed in more detail. The signal change in the raw data provides information about task-induced effects independent of the particular choice of control task. Six locations were chosen to cover the extent of the region of activation as shown in Fig. 3. The six locations and their coordinates are displayed in Fig. 4. For each peak, the percentage signal change in the corresponding voxel of the original

\footnotetext{
${ }^{4}$ The activation of components of the mesofrontal dopaminergic pathway on the left side, i.e., the ventral tegmental and retrorubral area together with the caudate nucleus/nucleus accumbens, is consistent with the frontomedian cortical activation (Williams and Goldman-Rakic, 1998). However, a more thorough discussion of this issue is beyond the scope of this article.
}

image (size $27 \mathrm{~mm}^{3}$ ) was separately averaged for each participant and each condition. Figure 5 displays the means of the resulting values for each of the four conditions, across a window of $11 \mathrm{~s}$, starting $1 \mathrm{~s}$ before the offset of the target sentences. The graphs clearly show that for all but the most ventral region (BA 10), the sentences elicited a positive-going curve. Mor eover, for these five peaks, the condition with the largest increase in the BOLD response was the coherent condition during Part $A$. In contrast, the coherence effect in BA 10 (Talairach coordinates [ -452 10]) was apparently due to a negative-going curve in the incoherent condition.

To further ensure that that the activations were statistically generalizable and stable across subjects, an additional region-of-interest (ROI) analysis was

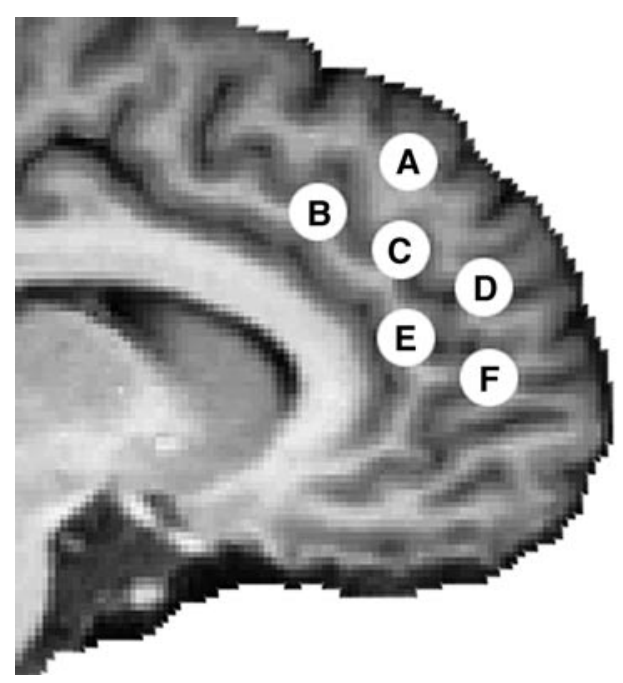

FIG. 4. Six regions of interest in FMC. 
A

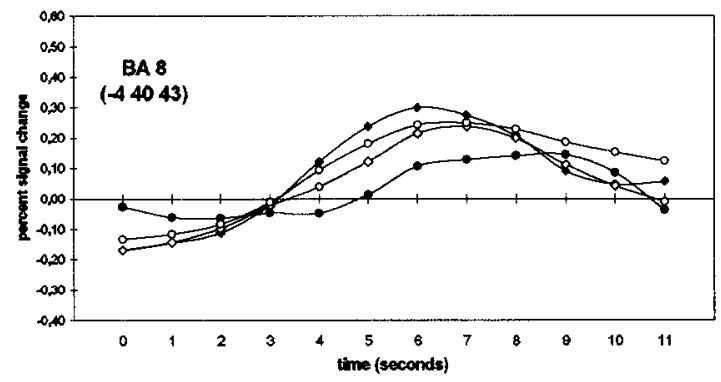

C

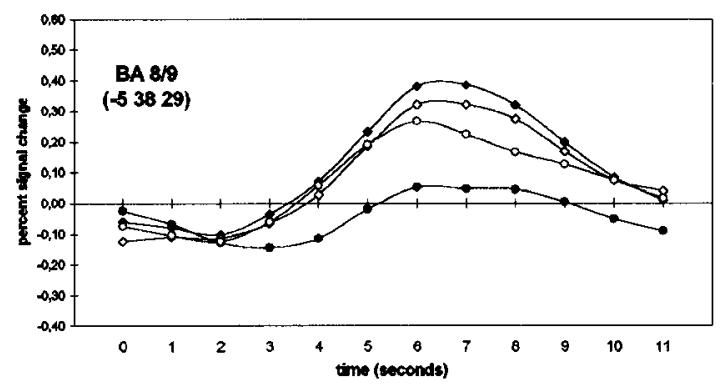

E

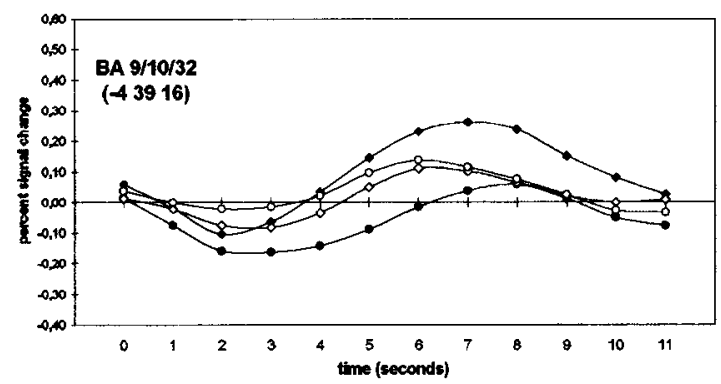

B

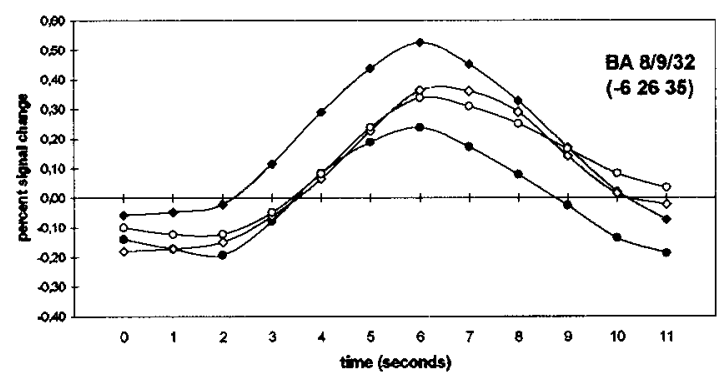

D

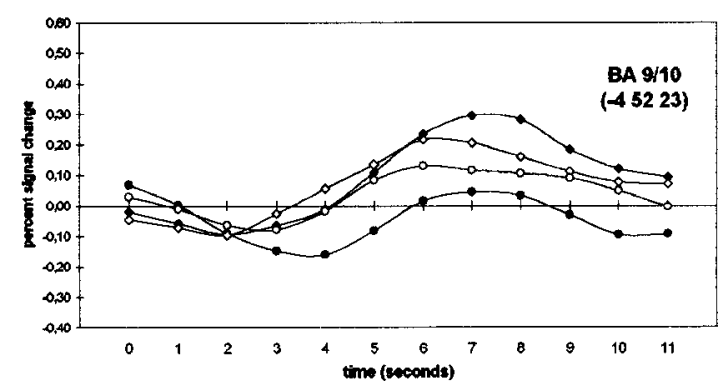

F

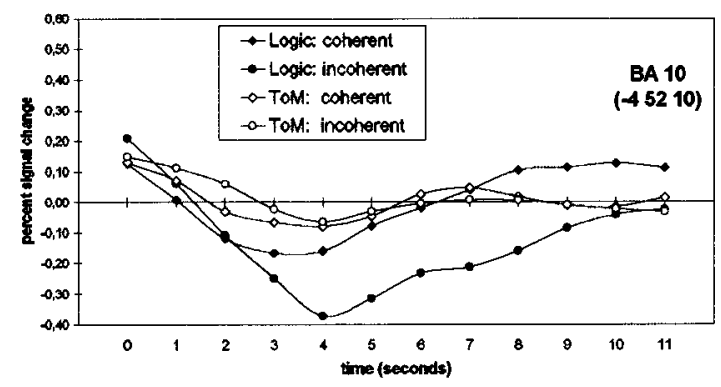

FIG. 5. Time course of activation in six frontomedian regions of interest (cf. Fig. 4). Shown is the percentage signal change for both parts of the experiment as a function of condition. The plots are time-locked at $1 \mathrm{~s}$ before the offset of the target sentences, which corresponds to the time locking used in the modeling of the event-related fMRI analysis.

conducted. Following the method proposed by Bosch (2000), Z maps were calculated for each condition and each participant, and nonlinearly normalized as described above. The peaks for six ROIs considered were identical to those taken for the time course diagrams. In a spherical region with a radius of $6 \mathrm{~mm}$ around the peak, the $Z$ values were averaged. Figure 6 shows the resulting means across participants, as a function of $\mathrm{ROI}$ and condition.

In the overall ANOVA with the within-subject factor ROI, Coherence, and Part, the main effects of ROI $(F(5,40)=12.0, P<0.0001)$ and Coherence $(F(1,8)=$ $5.5, p<0.05)$ were reliable. In contrast, there was no difference between the two parts of the experiment $(F(1,8)<1)$. Interactions between Coherence and Part $(F(1,8)=9.4, P<0.05)$ and between ROI and Part $(F(5,40)=2.7, P<0.05)$ once more confirm that the FMC activations varied systematically with the instructions and the materials. Most importantly, the

\section{Regions of Interest (FMC)}

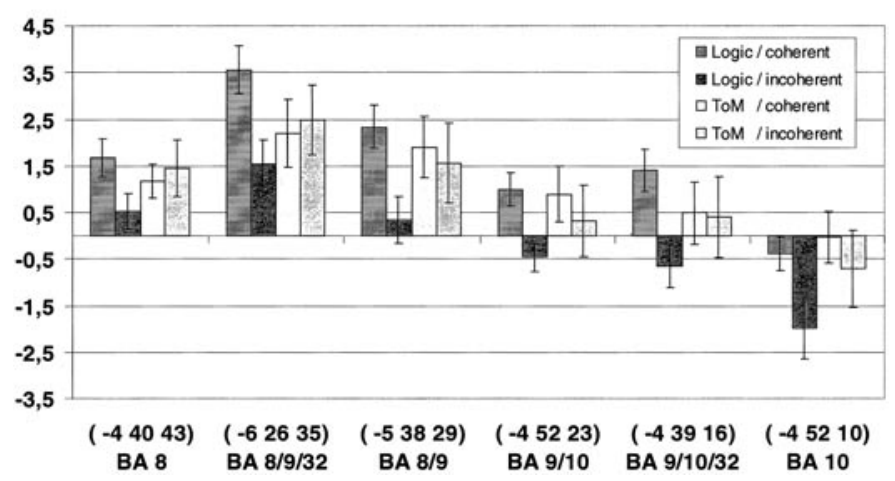

FIG. 6. Means and standard errors of the $z$ values of the six regions of interest, shown for both parts of the experiment as a function of coherence. The regions shown are A-F (cf. Fig. 4) from left to right. 
interaction between Coherence and ROI $(F(5,40)=2.7$, $\mathrm{P}<0.05)$ and the three-way interaction between Coherence, Part, and ROI indicated that the effects of coherence were reflected in different regions of the FMC, dependent on the type of processing $(F(3,24)=$ 7.4, $\mathrm{P}<0.01$ ). Planned pairwise comparisons, evaluating the effect of Coherence for each part and each ROI separately, yielded highly significant effects for all six ROIs during Part A ( $F$ 's $>13.0$, $P$ 's $<0.01$ ). During Part $B$, coherence had no corresponding effect ( $F$ 's $<$ $1)$. This analysis confirms that across the group of subjects, the effects of coherence during Part A were stable and generalizable.

\section{DISCUSSION}

In the present study we replicated the finding of the frontomedian cortex to be involved during Theory-ofMind processes. Incoherent as well as coherent trials elicited FMC activation during Part $B$, confirming that ToM processing was sufficient for engaging the $\mathrm{FMC}$. This result was also reflected in stronger $\mathrm{FMC}$ activation during Part B than during Part A. Supporting our hypotheses, the data also provided evidence for the claim that ToM processes are not necessary for activating this region, but that they are an instantiation of a more general cognitive process subserved by the FMC. During Part A the comprehension of coherent, plausible sentence pairs elicited frontomedian activation even though ToM processes were minimized. This influence of coherence during Part A only, but not during Part $B$, was also confirmed in a reliable interaction.

The behavioral data confirmed that the participants heeded the instructions; i.e., they used different processing strategies for the two parts of the experiment. Most importantly, the responses mostly coincided with the coherence of the sentence pairs during the Logic instructions. Thus, the comparison between coherent and incoherent trials tapped similar processes as in the previous study (Ferstl and von Cramon, 2001). Furthermore, it took longer to make a decision about whether the sentence pair described a comprehensible behavior (Part B) than about whether there was a logical connection (Part A). During the debriefing, most participants' self-reports confirmed that they attempted to identify with the protagonists. These results show that the different instructions for the sentence pairs with and without human protagonists had the intended effect of excluding ToM processes in Part $A$ and inducing them in Part B.

The activations found in the contrast of all language trials against the control task replicate many previous studies on language processing in context (e.g., Mazoyer et al., 1993; Ferstl and von Cramon, 2001). In particular, the bilateral temporal activation, including anterior and posterior parts of the superior temporal sulcus, as well as the temporoparietal junction area, was expected. The absence of a larger left-sided lateral prefrontal involvement in this contrast was probably due to the auditory presentation, in which both the language and the pseudo-word trials shared phonological processes. In the following, we focus on an interpretation of the two regions in the median wall of the left hemisphere.

It has been proposed that frontomedian regions are active during rest or during less attention-demanding tasks, and that more taxing cognitive processes yield deactivation or attenuation of these regions (Gusnard et al., 2001, Raichle et al., 2001). Gusnard et al. (2001) provided evidence for the argument that more ventral regions follow this pattern indeed (BA11), but that in their study, the more dorsal regions (BA 8/9/32) were positively activated by self-referential processing (i.e., a pleasantness judgment). In our study, we could show for six peaks of activation-differentially sensitive to the experimental condition-that a similar dissociation holds. In five of the six regions of interest, the BOLD contrast increased for the coherent conditions, whereas in the most ventral area, it decreased for the incoherent condition. As Binder et al. (1999) argued, the FMC activation found during rest conditions possibly reflects self-guided thought processes in the absence of a task. The fact that, in our study, activation increased and that these increases were dependent on the experimental condition confirms that the FMC plays a role not only as an area taking part in the representation of a "default state" (Raichle et al., 2001), but also during the performance of tasks requiring a self-guided, nonautomatic cognitive process, such as ToM and coherence tasks.

Other influential proposals for a specific function of the FMC, besides ToM, include affective processing (Greene et al., 2001; Lane et al., 1997; see also Maddock, 2001, for the proposal of the retrosplenial cortex/ PCC being involved in emotion processing). Raichle et al. (2001; see also Simpson et al., 2001a,b) see the precuneus as a region constantly monitoring the perceptual input, and the FMC as assessing the emotional significance of the external stimuli. The evidence for these proposals comes from the observation of FMC activation during tasks presumably eliciting emotions. The interpretations of the results are less than conclusive, though. For instance, Greene et al. (2001) used a moral judgment on complex moral dilemmas. Thus, the decision task required a variety of subprocesses, including the comprehension of the different texts and the decision taking into account the persons' value systems. It is not obvious that the emotional content was the crucial factor leading to FMC activation, rather than, let's say, inferences based on episodic memory, the ambiguity and uncertainty of the decision, or the more complex problem-solving process. Moreover, the data of the present study show that FMC activation was elicited by a condition in which 
emotional processes might play a supporting role at best. Of course, it is al ways possible for one experimental condition to be accompanied by stronger emotional involvement than another (see Simpson et al., 2001 a,b, for a similar argument), but it is not clear why this should be the case for the processing of coherent as compared with incoherent sentence pairs with emotionally neutral content.

A second proposal links the observed activations to self-referential processing (Gusnard et al., 2001). Although this concept is closely related, and not easily separable from the affect account (for instance, Lane et al., 1997, studying emotional processes, used an almost identical pleasantness judgment task as Gusnard et al., 2001, studying self-referential processing), it focuses more on the personal relevance of the to-beprocessed stimuli than on their emotional content. Vogeley et al. (2001) recently reported an experiment in which the self-relevance or self-perspective was systematically varied. The results showed that the FMC was engaged during both ToM and self-relevant processing. Zysset et al. (2002) indirectly manipulated self-reference in a study concerned with evaluative judgments. When the verification of factual statements, related to self-referential, episodic memories, was compared with that of semantic, general world knowledge statements, a very similar pattern of $\mathrm{FMC/}$ PCC activation was found as in the present study. Interestingly, the FMC activation was even stronger when an evaluative component was added, whereas the reverse was true for the PCC activation. These results indicate that the $\mathrm{PCC}$ activation is modulated by retrieval processes (cf. Krause et al., 1999) whereas the FMC activation increases with the inference demands of the task, over and above the self-referential nature of the stimuli. The data reported here confirm that self-reference, as specifically induced by the ToM instructions during Part $B$, leads to strong involvement of the FMC/PCC regions. However, as pointed out before, self-referentiality of the stimulus content is not necessary for eliciting this activation, because it was also observed for coherent trials during Part $A$.

To delineate the role of the FMC, not only for coherence and ToM processes, we postulate a more general common component shared by the multitude of other tasks shown to engage this brain region. How can we describe this underlying function? Neuropsychological descriptions of patients with ischemic infarctions in the cortical territory of the anterior cerebral artery focus on changes in drive and volition (Marin, 1991). Patients with bilateral lesions suffer from akinetic mutism, characterized by a complete lack of mental animation (Damasio, 1999). Even in unilateral cases, patients often show a lack of ideas, they fail to initiate actions, or they neglect to carry out plans. With respect to language, patients with left-sided lesions have a reduction of spontaneous speech. Based on these obser- vations was our previous interpretation of the FMC being involved whenever the initiation and maintenance of nonautomatic cognitive processes are required, independent of the domain and independent of the content (Ferstl and von Cramon, 2001). This assumption fits very well with the adaptive coding model (Duncan, 2001), postulating that a fundamental principle of prefrontal cortex might be its potential to be driven by many different kinds of input.

We now go one step further and link this domainindependent component to a concept from philosophy. Metzinger (2000) described a component of subjective experience, the so-called self-model, as a "transient computational module, episodically activated by the system in order to regulate its interaction with the environment" (p. 290). Not to be intermixed with the self-concept, encompassing the knowledge about the self, the self-model is continuously updated by integrating external and internal information in a dynamic way. Thus, the engagement of the self-model is dependent on both the stimulus features and the cognitive, motivational, and volitional status of the system. Speculative at this point, the self-model seems a promising account for the domain-general functionality of the FMC (see also Vogeley et al., 1999).

Researchers are just beginning to collect data directly targeting FMC functionality and to develop theoretical accounts thereof (e.g., Gusnard et al., 2001). Thus, a falsification of our still underspecified proposal seems difficult. Any experimental task requires some integration of the internal world with the external stimulus, with the focus being shifted from one to the other by both task requirements and stimulus properties. And indeed, FMC activation has been shown to be a matter of degree, rather than all-or-none (e.g., Castelli et al ., 2000; Greene et al., 2001; Zysset et al., 2002), and it cannot directly be predicted by stimulus complexity or task difficulty. Even during rest conditions FMC activation has been observed-similar to that elicited by knowledge-based semantic processing-indicating the initiation and maintenance of self-guided thought (Binder et al., 1999). Consequently, conclusions about which tasks will and which tasks will not engage the FMC are highly dependent on the control task. For instance, the rather demanding memory task used by Fletcher et al. (1995; Gallagher et al., 2000) as a control task ("unrelated sentences") requires selfguided encoding strategies and is thus predicted to lead to comparable FMC activation as the comprehension of the "physical stories"-a condition whose inference requirements make F MC engagement likely. F urther research is needed for directly investigating these issues. We are confident that the proposal sketched above will prove useful for generating testable hypotheses which will, in turn, aid in refining the theoretical understanding of the functional neuroanatomy of the FMC. 


\section{CONCLUSIONS}

In this study we presented evidence for a domainindependent, general-purpose functionality of the FMC. Based on previous observations of involvement of FMC during coherence processes as well as during ToM, emotional, or self-referential processing, we designed a study in which coherence and ToM were independently varied. The result of activation of both the FMC and an accompanying region in the PCC TOM processing, but also whenever sentence pairs were coherently related, suggests that inference processes are sufficient for engaging median cortical structures. We believe that the function of the FMC includes the initiation and maintenance of nonautomatic cognitive processes. However, further research is needed to obtain a better understanding of this brain area's functionality. A future theory must include a more thorough delineation of the subdivisions of FMC, as well as a consideration of volitional and motivational aspects of cognition.

\section{REFERENCES}

Baron-Cohen, S., Leslie, A. M., and Frith, U. 1985. Does the autistic child have a 'theory of mind'? Cognition 21: 37-46.

Baron-Cohen, S., Ring, H. A., Wheelwright, S., Bullmore, E. T., Brammer, M. J ., Simmons, A., and Williams, S. C. R. 1999. Social intelligence in the normal and autistic brain: An fMRI study. Eur. J . Neurosci. 11: 1891-1898.

Binder, J . R., Frost, J . A., Hammeke, T. A., Bellgowan, P. S. F., Rao, S. M., and Cox, R. W. 1999. Conceptual processing during the conscious resting state: A functional MRI study. J . Cogn. Neurosci. 11: 80-93.

Bosch, V. 2000. Statistical analysis of multi-subject fMRI data: The assessment of focal activations. J . Magn. Reson. Imaging 11: 6164.

Bottini, G., Corcoran, R., Sterzi, R., Paulesu, E., Schenone, P., Scarpa, P., Frackowiak, R. S. J ., and Frith, C. D. 1994. The role of the right hemisphere in the interpretation of figurative aspects of language: A positron emission tomography activation study. Brain 117: 1241-1253.

Brothers, L. 1990. The social brain: A project for integrating primate behaviour and neurophysiology in a new domain. Concepts Neurosci. 1: 27-51.

Brunet, E., Sarfati, Y., Hardy-Baylé, M. C., and Decety, J . 2000. A PET investigation of the attribution of intentions with a nonverbal task. Neurol mage 11: 157-166.

Castelli, F., Happé, F., Frith, U., and Frith, C. 2000. Movement and mind: A functional imaging study of perception and interpretation of complex intentional movement patterns. Neurol mage 12: 314325.

Channon, S., and Crawford, S. 2000. The effects of anterior lesions on performance on a story comprehension test: Left anterior impairment on a theory of mind-type task. Neuropsychologia 38: 10061017.

Damasio, A. R. 1999. The Feeling of What Happens: Body and Emotion in the Making of Consciousness. Harcourt Brace, New York.

Duncan, J . 2001. An adaptive coding model of neural funciton in prefrontal cortex. Nat. Rev. Neurosci. 2: 820-829.
Ferstl, E. C., and von Cramon, D. Y. 2001. The role of coherence and cohesion in text comprehension: An event-related fMRI study. Cogn. Brain Res. 11: 325-340.

Ferstl, E. C., Guthke, T., and von Cramon, D. Y. 1999. Change of perspective in discourse comprehension: Encoding and retrieval processes after brain injury. Brain Lang. 70: 385- 420.

Fine, C., Lumsden, J ., and Blair, R. J . R. 2001. Dissociation between 'theory of mind' and executive functions in a patient with early left amygdala damage. Brain 124: 287-298.

Fletcher, P. C., Happe, F., Frith, U., Baker, S. C., Dolan, R. J ., Frackowiak, R. S. J ., and Frith, C. D. 1995. Other minds in the brain: A functional imaging study of "theory of mind" in story comprehension. Cognition 57: 109-128.

Friston, K. J . 1994. Statistical parametric maps in functional imaging: A general linear approach. Hum. Brain Mapp. 2: 189-210.

Frith, C. D., and F rith, U. 1999. Interacting minds, a biological basis. Science 286: 1692-1695.

Frith, U. 1989. Autism: Explaining the Enigma. Blackwell, Oxford.

Gallagher, H. L., Happé, F., Brunswick, N., Fletcher, P. C., Frith, U., and Frith, C. D. 2000. Reading the mind in cartoons and stories: An fMRI study of 'theory of mind' in verbal and nonverbal tasks. Neuropsychologia 38: 11-21.

Goel, V., Gold, B., Kapur, S., and Houle, S. 1997. The seats of reason? An imaging study of deductive and inductive reasoning. NeuroReport 8: 1305-1310.

Goel, V., Grafman, J., Sadato, N., and Hallett, M. 1995. Modeling other minds. NeuroReport 6: 1741-1746.

Greene, J . D., Sommerville, R. B., Nystrom, L. E., Darley, J . M., and Cohen, J . D. 2001. An fMRI investigation of emotional engagement in moral judgment. Science 293: 2105-2108.

Gusnard, D. A., Akbudak, E., Shulman, G. L., and Raichle, M. E. 2001. Medial prefrontal cortex and self-referential mental activity: Relation to a default mode of brain function. Proc. Natl. Acad. Sci. USA 98: 4259- 4264.

Happé, F. 2000. Parts and wholes, meaning and minds: Central coherence and its relation to theory of mind. In Understanding Other Minds: Perspectives from Developmental Cognitive Neuroscience (S. Baron-Cohen, H. Tager-Flusberg, and D. J. Cohen, Eds.), 2nd ed., pp. 203-221. Oxford Univ. Press, Oxford.

Happé, F., Brownell, H. H., and Winner, E. 1999. Acquired theory of mind' impairments following stroke. Cognition 70: 211-240.

Happé, F., Ehlers, S., Fletcher, P., Frith, U., J ohansson, M., Gillberg, C. Dolan, R., Frackowiak, R., and Frith, C. 1996. 'Theory of mind' in the brain: Evidence from a PET scan study of Asperger syndrome. NeuroReport 8: 197-201.

J olliffe, T., and Baron-Cohen, S. 1999. A test of central coherence theory: Linguistic processing in high-functioning adults with autism or Asperger syndrome: Is local coherence impaired? Cognition 71: 149-185.

J olliffe, T., and Baron-Cohen, S. 2000. Linguistic processing in highfunctioning adults with autism of Asperger's syndrome: Is global coherence impaired? Psychol. Med. 30: 1169-1187.

Kintsch, W. 1998. Comprehension: A Paradigm for Cognition. Cambridge University Press, Cambridge.

Krause, B. J ., Schmidt, D., Mottaghy, F. M., Taylor, J ., Halsband, U., Herzog, H., Tellmann, L., and Müller-Gärtner, H.-W. 1999. Episodic retrieval activates the precuneus irrespective of the imagery content of word pair associates: A PET-study. Brain 122: 255-263.

Lane, R. D., Fink, G. R., Chau, P. M. L., and Dolan, R. J . 1997. Neural activation during selective attention to subjective emotional responses. NeuroReport 8: 3969-3972.

Lohmann, G. 1998. Volumetric Image Analysis. Wiley, Chichester. Lohmann, G., Müller, K., Bosch, V., Mentzel, H., Hessler, S., Chen, L., and von Cramon, D. Y. 2001. Lipsia: A new software system for 
the evaluation of functional magnetic resonance images of the human brain. Comput. Med. Imaging Graphics 25: 449- 457.

Maddock, R. J . 2001. The retrosplenial cortex and emotion: New insights from functional neuroimaging of the human brain. Trends Neurosci. 22: 310-316.

Maguire, E. A., Frith, C. D., and Morris, R. G. M. 1999. The functional neuroanatomy of comprehension and memory: The importance of prior knowledge. Brain 122: 1839-1850.

Marin, R. W. 1991. Apathy: A neuropsychiatric syndrome. J . Neuropsychiatry Clin. Neurosci. 3: 243-254.

Mazoyer, B. M., Tzourio, N., Frak, V., Syrota, A., Murayama, N., Levrier, O., Salamon, G., Dehaene, S., Cohen, L., and Mehler, J . 1993. The cortical representation of speech. J . Cogn. Neurosci. 5: 467- 479.

Metzinger, T. 2000. The subjectivity of subjective experience: A representationalist analysis of the first-person perspective. In Neural Correlates of Consciousness: Empirical and Conceptual Questions (T. Metzinger, Ed.), pp. 285-306. MIT Press, Cambridge, MA.

Nichelli, P., Grafman, J., Pietrini, P., Clark, K., Lee, K. Y., and Miletich, R. 1995. Where the brain appreciates the moral of a story. NeuroReport 6: 2309-2313.

Norris, D. G. 2000. Reduced power multi-slice MDEFT imaging. J . Magn. Reson. Imaging 11: 445- 451.

Premack, D., and Woodruff, G. 1978. Does the chimpanzee have a theory of mind? Behav. Brain Sci. 1: 516-526.

Raichle, M. E., MacLeod, A. M., Snyder, A. Z., Powers, W. J ., Gusnard, D. A., and Shulman, G. L. 2001. A default mode of brain function. Proc. Nat. Acad. Sci. USA 98: 676-682.

Rowe, A. D., Bullock, P. R., Polkey, C. E., and Morris, R. G. 2001. Theory of mind' impairments and their relationship to executive functioning following frontal lobe excisions. Brain 124: 600-616.

Siegal, M., Carrington, J ., and Radel, M. 1996. Theory of mind and pragmatic understanding following right hemisphere damage. Brain Lang. 53: 40-50.

Simpson, J . R., Drevets, W. C., Snyder, A. Z., Gusnard, D. A., and Raichle, M. E. 2001a. Emotion-induced changes in human medial prefrontal cortex: II. During anticipatory anxiety. Proc. Natl. Acad. Sci. USA 98: 688-693.

Simpson, J . R., Snyder, A. Z., Gusnard, D. A., and Raichle, M. E. 2001b. Emotion-induced changes in human medial prefrontal cortex: I. During cognitive task performance. Proc. Natl. Acad. Sci. 98: 683- 687.

Stone, V. E., Baron-Cohen, S., and Knight, R. T. 1998. Frontal lobe contributions to theory of mind. J . Cogni. Neurosci. 10: 640- 656.

Stuss, D. T., Gallup, G. G., and Alexander, M. P. 2001. The frontal Iobes are necessary for 'theory of mind'. Brain 124: 279-286.

Talairach, J ., and Tournoux, P. 1988. Coplanar Stereotaxic Atlas of the Human Brain. Thieme, New York.

Thirion, J. P. 1998. Image matching as a diffusion process: An analogy with Maxwell's demons. Med. Image Analy. 2: 243-260.

Ugurbil, K., Garwood, M., Ellermann, J., Hendrich, K., Hinke, R., Hu, X., Kim, S.-G., Menon, R., Merkle, H., Ogawa, S., and Salmi, R. 1993. I maging at high magnetic fields: I nitial experiences at 4T. Magn. Reson. Q. 9: 259.

Vogeley, K., Bussfeld, P., Newen, A., Herrmann, S., Happé, F., Falkai, P., Maier, W., Shah, N. J ., Fink, G. R., and Zilles, K. 2001. Mind reading: Neural mechanisms of theory of mind and selfperspective. Neurol mage 14: 170-181.

Vogeley, K., Kurthen, M., Falkai, P., and Maier, W. 1999. Essential functions of the human sellf model are implemented in the prefrontal cortex. Consciousness Cognition 8: 343-363.

Wellman, H. M. 1993. Early understanding of mind: The normal case. In Understanding Other Minds: Perspectives from Autism (S. Baron-Cohen, H. Tager-Flusberg, and D. J . Cohen, Eds.), pp. 1039. Oxford Univ. Press, Oxford.

Williams, S. M., and Goldman-Rakic, P. S. 1998. Widespread origin of the primate mesofrontal dopamine system. Cereb. Cortex 8: 321-345.

Zysset, S., Huber, O., Ferstl, E. C., and von Cramon, D. Y. 2002. The anterior fronto-median cortex and evaluative judgment: An fMRI study. Neurol mage 15: 983-991. 